\title{
Energy Detection Based Spectrum Sensing Technique for Software Defined Radio
}

\author{
Tapan K Das , Yashaswini Sharma \\ ${ }^{1}$ Retd. Maj.Gen (VSM, Associate Professor), Department of Electronics and Communication Engineering, JECRC University, Jaipur, \\ Rajasthan, India
}

${ }^{2}$ M.Tech Scholar, Department of Electronics and Communication Engineering, JECRC University, Jaipur, Rajasthan, India

\begin{abstract}
A lot of hardware components are used to make a conventional radio communication system, the complexity of these hardware can be reduced with help of Software Defined Radio (SDR) which is a form of wireless communication. Software Defined Radio implemented system allows us to make alteration in it or operate it with help of a software, thus making the system flexible and reconfigurable. The system changes from single mode or single band to multi-mode, multi-band as we can change the functionality and parameters of the system with the help of a software. The hardware components of a traditional radio communication system such as converters, modulator, demodulator, detector, filter, etc, make the platform cost very high and increases the complexity of a system. Software Defined Radio helps in excluding all the hardware from the system as it replaces them by pure software. In SDR modulation plays a very vital role as different modulation techniques are very important part of wireless technology and radio communication. This paper discusses about the amplitude modulation in brief and also discusses the optimized utilization of spectrum resources with help of software defined radio.
\end{abstract}

Keywords: Software Defined Radio, Power Spectral Density, Spectrum Hole, Primary User, Secondary User

\section{Introduction}

Amplitude Modulation transmits information from one system to another through radio carrier wave and is widely used in electronic communication. In amplitude modulation, in line with variations in intensity of sound wave, the amplitude or signal strength of carrier wave is varied. In this way overall amplitude or envelope of carrier is modulated. A Canadian engineer named Reginald Fesseden was the first person who recorded AM in 1900. In the process he placed a carbon microphone in the antenna lead by which amplitude modulation was done through continuous spark transmission. Nowadays, wireless applications require spectrum resources. Each new service is provided with its own frequency block. This pre-decided spectrum allotment to users lead to wastage of spectrum, as huge portion of spectrum is not productively used; some of it is used to a limited extent, while some of it is left completely unused. The unlicensed users are not allowed to access the licensed spectrum as it is restricted by current government policies; as a result, they use interference - prone and heavily populated frequency bands, which may be already in use. Thus in those bands there is huge spectrum scarcity problem. The major government agency such as Telecom Regulation Authority of India (TRAI) is responsible for maintaining the radio spectrum as it has been a limited resource. It is observed that some of the frequency bands in the spectrum are unutilized for some time, most of the frequency bands are partially occupied and some of the bands are heavily used. This observation is done by scanning the radio spectrum in which the revenue rich urban areas are included.

This paper discusses about the project work in which a Software Defined Radio (SDR) is developed which will help in improving the utilization of spectrum by allowing secondary user to share the spectrum with the primary users or if the radio spectrum is not in use by them then to borrow it from primary users. Software Defined radio is acquainted with the radio frequency environment, the communication parameters (such as modulation type, carrier frequency, transmission power and bandwidth) are chosen appropriately to make the optimized utilization of the spectrum and adapt transmission and reception accordingly. The SDR need to fill in the available spectrum holes by allotting these gaps to new upcoming users and hence can avoid the harmful interference to licensed user. For this, the Software Defined radio senses the spectrum continuously in order to detect the re-appearance of the primary user and once the primary user is detected, SDR should pull out itself from the spectrum gap it is using; this will help in avoiding the interference. While communication there may be variable propagation environment and also secondary users may generate interference. Thus accomplishment of this task becomes very difficult because of such environment conditions and also different primary users will be employed with no. of parameters such as different modulation techniques, varying data rates and transmission powers. Communication system is a broad area and is collection of many different processes including modulation, conversion, amplification etc. Modulation is a very important and basic process of communication, a type of modulation such as amplitude modulation has a great significance in communication system, hence this technique is also performed with help of software defined radio.

\section{Literature Review}

1) In the paper "SDR - Implementation of low frequency trans-receiver on FPGA" by Sunil G Yankanchiand Mrs. Kavitha .D (2015), it is discussed that FPGA is the best suited platform to achieve reprogrammed radio components. For complex design systems of the radio, SDR (Software Defined Radio) is the basic element to be considered as it can be programmed again and again to perform many functions making it configurable. In this 


\section{International Journal of Science and Research (IJSR) \\ ISSN (Online): 2319-7064 \\ Index Copernicus Value (2015): 78.96 | Impact Factor (2015): 6.391}

project they have done the design implementation of low frequency transceiver using vitrex 5 device. System level models and designs are carried out using system generator.

2) In paper "Investigating Latency in GNU Software Radio with USRP Embedded Series SDR Platform" by Nguyen B.Truong and Chansu Yu (2013), it is studied that besides the advantages like re-configurability and flexibility, a SDR system has to face a critical challenge on real-time processing due to high jitter and latency. Thus, understanding latency in SDR systems is very important. The paper focuses on investigating the latency of a popular SDR platform - GNU Software Radio with a new Universal Software Radio Peripherals (USRP) device-USRP Embedded Series.

3) In paper "SDR Implementation for DCF77" by Filip Zaplata and Miroslav Kasal (2013), The DCF77 signal is used to carry out the information of precise time for long distances. Standard DCF77 receivers consist of AM long wave receiver circuit, connected to a data processing unit. The idea is to remove the analog demodulator and process the amplified RF signal also on the processor. Specialized AM radio chip then would be replaced by a simple amplifier, e.g. operational amplifier circuit.

4) In the paper "Spectrum Sensing Techniques in cognitive radio Networks: A survey" by Mansi Subhedar and Gajanan Birajdar (2011), survey of spectrum sensing techniques is presented. The challenges and issues involved in implementation of spectrum sensing techniques are discussed in detail giving comparative study of various methodologies.

5) In the paper "Optimized configurable architecture of modulation techniques for SDR applications" by Jignesh Oza et al.,(2010),Implementation of modulation techniques with the approach of optimizing the architecture is shown, it is implemented on FPGA and MATLAB, it shows how it helps in reducing the architecture of SDR. The paper describes modulation techniques FSK and QPSK when configured together yields max optimization with good features.

6) The paper "Mapping of the FFT on a Reconfigurable Architecture targeted to SDR Applications", by Fabio Garzia et al., (2009), describes the implementation of a FFT on a system based on a GP core and a reconfigurable coarse-grain accelerator. The entire system has been proto typed on an Altera Stratix II device. On the prototype a 1024-point FFT gives a 40X speed-up in comparison with the software implementation. The 1024-point FFT is executed.

\section{Concept of Software Defined Radio (SDR)}

In this project a Software Defined radio is developed as a form of wireless communication where it will perform the amplitude modulation and a transceiver is made to detect which of the channels are being used and which are not in use for communication and while avoiding the channels which are occupied, access through the unused channels.
Spectrum hole $(\mathrm{SH})$ is the un-utilized slot of spectrum or that spectrum band which can be accessed and utilized by unlicensed users. The spectrum holes are detected by sensing whether a primary signal is present or absent in particular band and if the primary user is absent then try to access them. The primary users utilize the spectrum band either at different time slots or in different geographic regions.

Spectrum holes can be contemplated as multidimensional regions within frequency, time and space and because of them we can make non- interfering or safe use of spectrum as these vacant bands represent absence of primary users. The main task for software defined radio systems is to be able to strongly sense when they are within such a spectrum hole and once found, they can make use of it. The concept of Spectrum Hole is depicted in Figure (1).

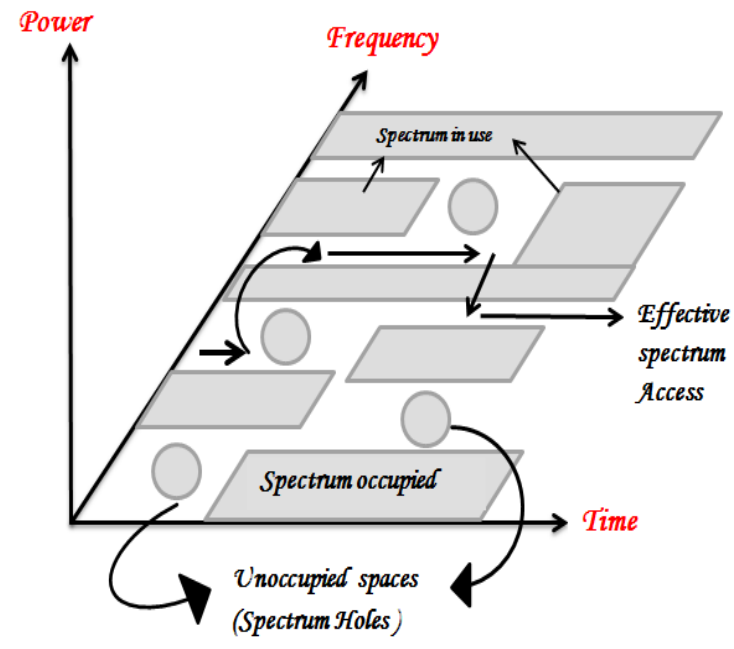

Figure 1: Illustration of Spectrum Hole

\subsection{Power Spectral Density}

The energy of the frequencies, whether the variations (energy) are strong or weak is depicted by the Power Spectral Density of the signal. In other words PSD characterises the frequency content of a signal which is estimated by Fast Fourier Transform (FFT), it also represents the distribution of power of a signal over the different frequencies. The integral of power spectral density or the average power is fed as input to energy detector which in output would determine the total energy of the signal.

\subsection{Energy detection}

Since long time, people are recommending methods to identify spectrum detection as well as for presence of signal transmission. This is done with FFT (Fast Fourier Transform) which helps in indicating the presence of licensed signal. The technique which uses this method is energy detection technique. This basically determines the strength of Power Spectral Density (PSD, which is defined as average power of the signal) in each frequency of the signal.

\subsection{Spectrum Hole}




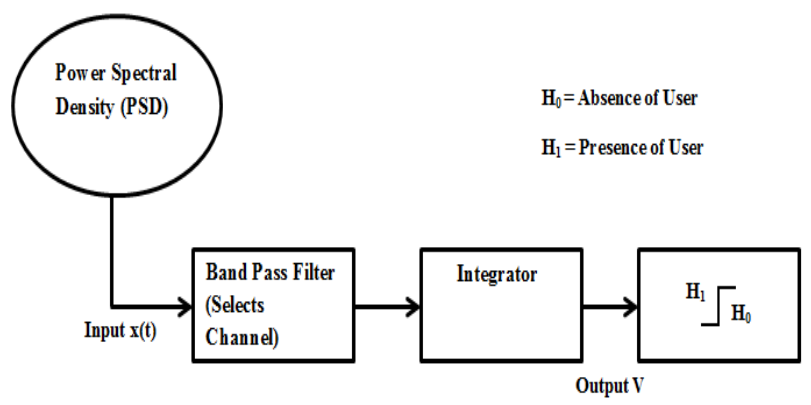

Figure 2: Energy Detection Technique

To limit the noise, Band Pass filter would filter the input signal $x(t)$ and will also select the channel of interest. Next in the diagram is the finite time integrator which will perform integration over time $\mathrm{T}$.

$x(t)=w(t)$, Ho (Primary or licensed User absent)

$x(t)=w(t)+h n(t)$, H1 (Primary or licensed User present)

Signal received by SDR user : $\mathbf{x}(\mathbf{t})$

Primary User's transmitted signal : $\mathbf{n}(\mathbf{t})$

Additive White Gaussian Noise (AWGN) with zero mean and variance $\sigma_{n}^{2}: \mathbf{w}(\mathbf{t})$

Amplitude Gain of the channel : $\mathbf{h}$

The strength of PSD determines the presence or absence of primary user. Primary user is present if the average power is high and if it is low, it indicates absence of primary user. This phenomenon is depicted with the hypothesis. $\mathrm{H} 0$ is a null hypothesis, which determines that there is no licensed user signal i.e. primary user is absent and $\mathrm{H} 1$ states that signal is transmitted, which means primary user is present . A pre- decided threshold value is compared with the final output of the energy detector and on the basis of that we get aware of the signal status. The output signal $\mathrm{V}$ from the integrator is

$$
V=1 / T \int_{t-T}^{t}|x(r)|^{2} d r
$$

Comparing this output signal to threshold value we get the presence or absence of signal.

\section{Project Work}

For the analysis, a message frequency, sampling frequency and 5 Carrier Frequency Bands for users are initialized first . Different values are given to different frequencies .

\subsection{In the first step}

AM modulates the data in the first step over the respective frequency bands. After that, all the modulated signals are added in adder to produce a transmitting signal.

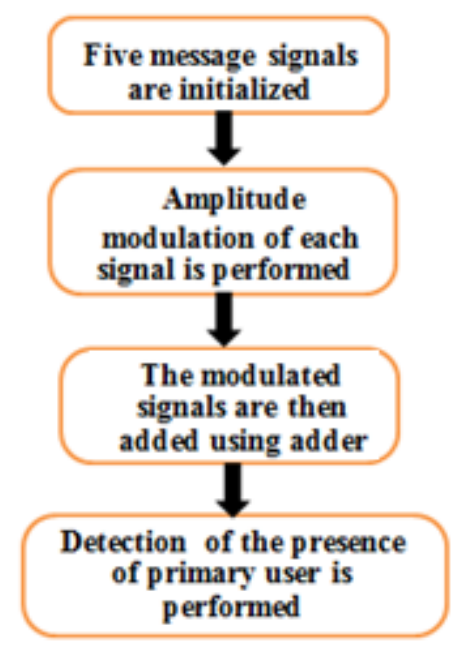

Figure 3: Depicting initial steps

\subsection{The second step includes}

- With help of Fast Fourier Transform (FFT) in MATLAB, the Power Spectral Density of the received signal is estimated using Periodogram.

- The average power in the signal over a frequency band is computed by taking the integral of PSD over that particular frequency band.

- If the integral of power spectral density i.e. the average power of the signal is high, it indicates the presence of primary user.

- Low Power Spectral Density (PSD) indicates absence of primary user.

- The first spectral hole is allotted to new user a soon as it arrives. If new users keep on arriving, the holes are allotted one by one accordingly.

- If primary user re- appears in the spectrum, any of the new user is asked to empty a specific slot if all the slots are engaged.

- We can analyse the channel characteristics by adding certain amount of noise and attenuation.

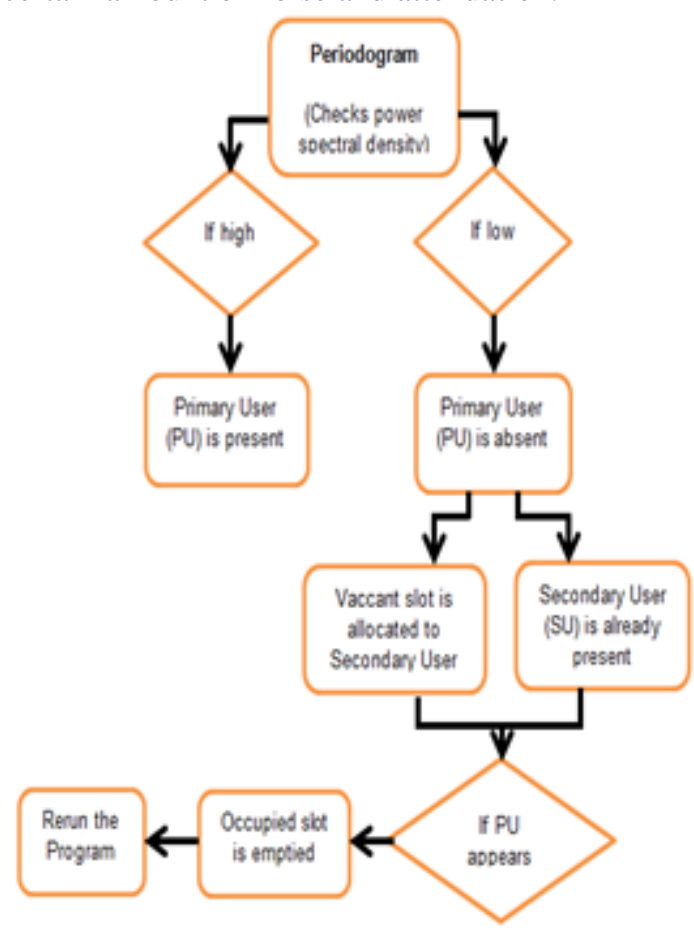

Figure 4: Second step flowchart 


\section{International Journal of Science and Research (IJSR) \\ ISSN (Online): 2319-7064}

Index Copernicus Value (2015): 78.96 | Impact Factor (2015): 6.391

\section{Results}

After performing Amplitude Modulation, the project leads to second step i.e. calculation of Power Spectral Density (PSD). The results are compared with predefined threshold value. This would provide us with the status of presence of primary user signal. The simulation is done for five signals. These signals will be having sampling frequency of $12 \mathrm{KHz}$ and carrier frequencies will be $1 \mathrm{KHz}, 2 \mathrm{KHz}, 3 \mathrm{KHz}, 4 \mathrm{KHz}$ and $5 \mathrm{KHz}$ respectively.

After calculating absence or presence of primary user, in this analysis, it has been taken that primary users at 3 slots i.e. second, third and fourth are absent. And at two slots i.e. $1^{\text {st }}$ and $5^{\text {th }}$ the primary users are present.

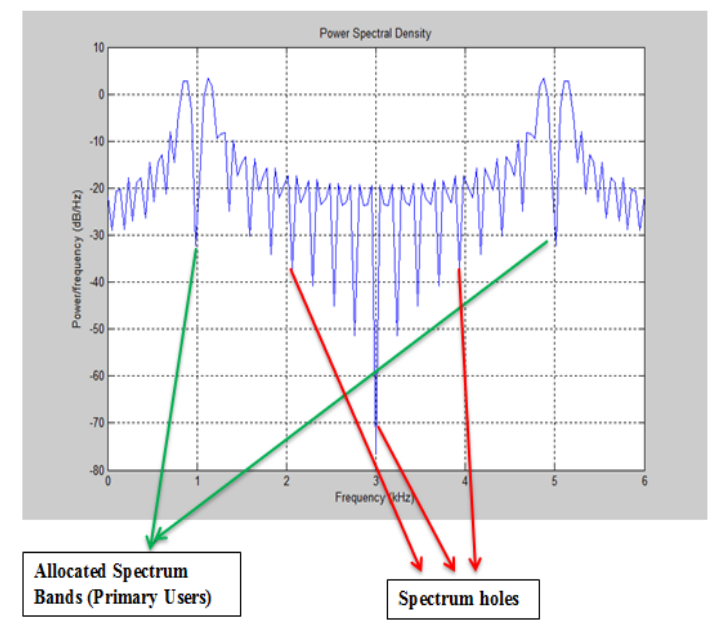

Figure 5: Adder Output

The graph gives the adder output. Green arrow bands indicate the occupied or used bands (1st and 5th), red ones are the unused bands ( $2 \mathrm{nd}, 3 \mathrm{rd}$, and $4 \mathrm{th}$ ). The amplitude or the peak value is between ( 15 to 30 ) $\mathrm{dB} / \mathrm{Hz}$ for the occupied spectral gaps or where the primary user is already present.After searching for the first available spectral gap (Spectrum Hole), the Software Defined Radio (SDR) system will automatically assign that hole to the secondary user (SU) in the spectrum. It is shown in the figure below that the first available spectral gap was occupied by the secondary user (SU) 1.

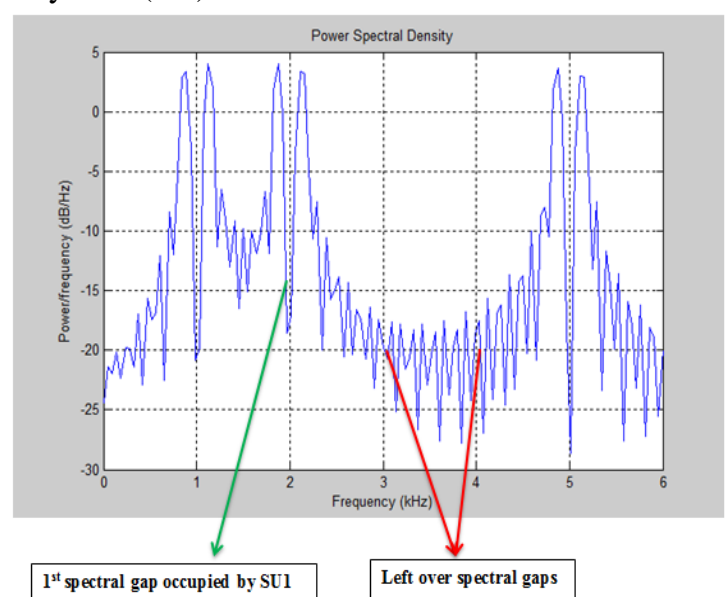

Figure 6: Secondary User 1(SU1) occupies 1st spectral gap

A greater dip in the peak indicated by the green arrow shows that secondary user has occupied that place.
As the first available spectrum hole was assigned to new user by system, in the same way the next available spectrum gaps would also be assigned one by one automatically to the upcoming users in spectrum. As soon as all the slots are allotted to the users, system will not entertain any other user and will be able to free up the spectral gap (slot) one by one. If the primary user reappears and the system needs an empty slot, it will eradicate the data in the first spectral gap by asking the secondary user to leave the hole and make it ready for the next upcoming assignment. Graph after all the spectrum holes are occupied one by one is as shown.

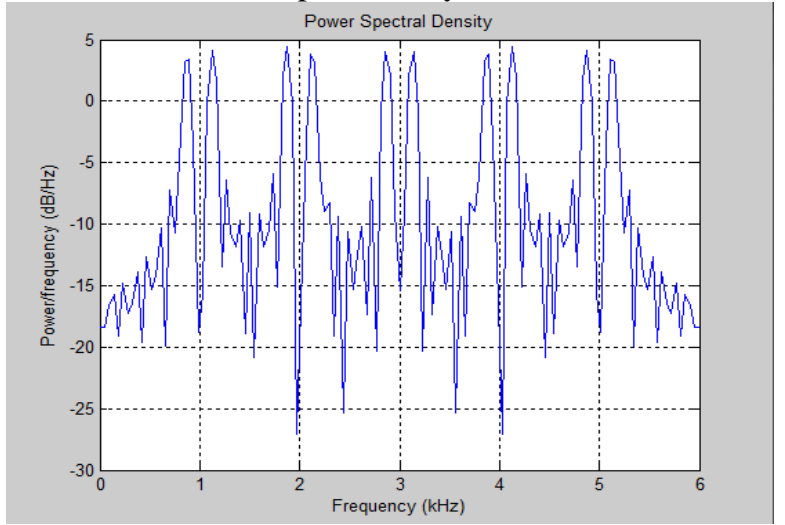

Figure 7: All spectral holes are occupied

The channel characteristics can be analysed by adding noise and attenuation parameter. Taking two values of each.

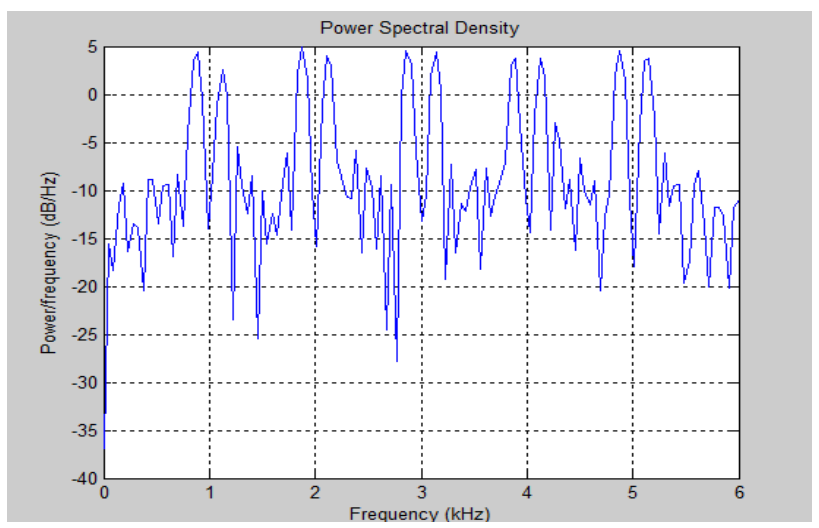

Figure 8: First the Signal to Noise Ratio (SNR) is $10 \mathrm{~dB}$

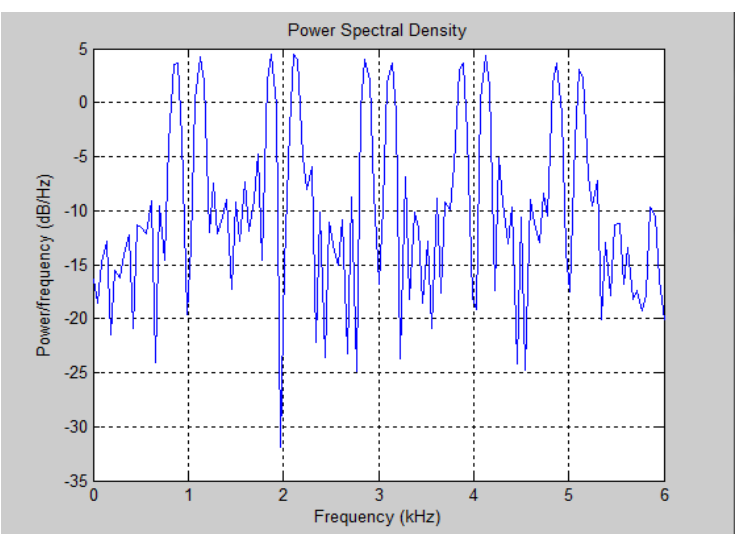

Figure 9: For the second case taking the SNR to be $15 \mathrm{~dB}$

It can be observed through the graphs obtained that as the SNR increases, the disturbance in the spectrum decreases. This makes it obvious that the probability of the error in the received signal is increased due to noise. If we take the 


\section{International Journal of Science and Research (IJSR) \\ ISSN (Online): 2319-7064}

Index Copernicus Value (2015): 78.96 | Impact Factor (2015): 6.391

attenuation percentage values as $20 \%$ and $40 \%$ we get the following results. It is observed that as the attenuation increases, the signal peaks reduce proportionately. This reduces the signal power which results in weakening of the proper signal reception.

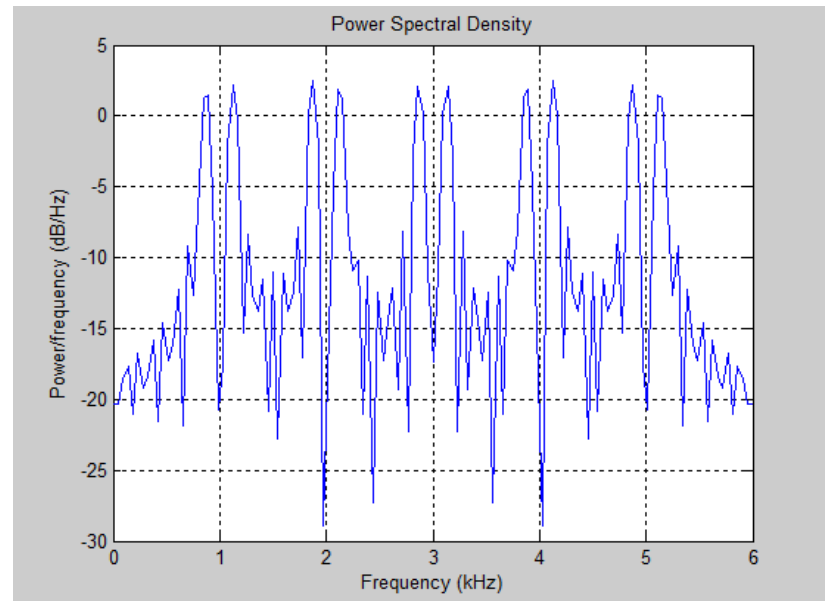

Figure 10: Attenuation $=20 \%$

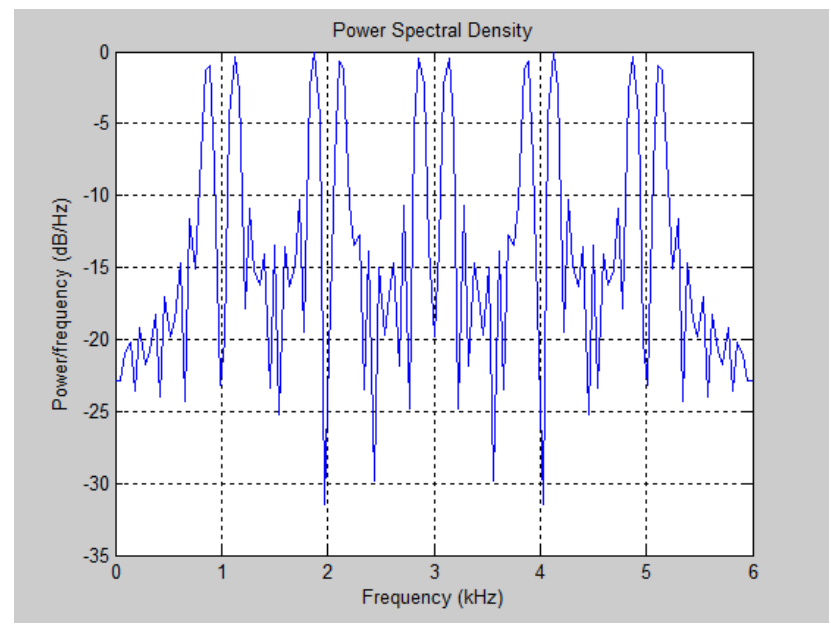

Figure 11: Attenuation $=40 \%$

\section{Conclusion}

The idea in this project was to perform the amplitude modulation using SDR and take decisions on the basis of power spectral density (PSD) of the channel which can be used to search the available spectral gaps/holes .These available spectrum holes can be provided to new incoming users i.e. Secondary Users (SU) which will thus improve the entire channel's throughput as now the spectrum band is properly utilized. In this work the method of energy detection for spectrum sensing is done using FFT within the specified frequency band with help of MATLAB. It is successfully demonstrated in simulation results that Software Defined Radio can work in a very dynamic manner with changing frequency band from one to another. The values for the Signal to Noise ratio (SNR) are taken as $10 \mathrm{~dB}, 15 \mathrm{~dB}$ and Attenuation percentages $20 \%$ and $40 \%$ has been used to show the simulation results.

\section{References}

[1] Yankanchi S.G. and D.Kavitha (2015), "SDR Implementation of low frequency trans-receiver on FPGA", International Journal of Engineering Research and Technology (IJERT), ISSN: 2278-0181, Volume-4, Issue-05.

[2] Truong N.B. and Yu Chansu (2013), "Investigating Latency in GNU Software Radio with USRP Embedded Series SDR Platform", IEEE Eighth International Conference on Broadband, Wireless Computing, Communication and Applications.

[3] Zaplata F. and Kasal M. (2013), "SDR Implementation for DCF77”, IEEE 23th Conference Radioelektronika, Pardubice, Czech Republic.

[4] Subhedar M. and Birajdar G. (2011), "Spectrum Sensing Techniques in Cognitive Radio Networks: A survey" International Journal of Next-Generation Networks (IJNGN), Vol.3, No.2.

[5] Oza J., Patel Y. , Trivedi P., Ranpura N., Patel Z., Dalal U., Jani R. and S.R. Vijay (2010), "Optimized configurable architecture of modulation techniques for SDR applications", International Conference on Computer and Communication Engineering (ICCCE).

[6] Garzia F., Airoldi R., Nurmi J., Giliberto C. and Brunelli C. (2009), "Mapping of the FFT on a Reconfigurable Architecture targeted to SDR Applications" IEEE 978-1-4244-4467.

\section{Author Profile}

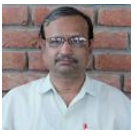

Major General Tapan Kumar Das, VSM (Retd.) is working as Associate Professor in the department of Electronics and Communication Engineering at JECRC University, Jaipur, Rajasthan. He completed his B.Tech in Telecommunication and IT, M.E in Communication Engineering from Jadavpur University and PG Diploma in International Business from Pondicherry University. He attended many international and national conferences. His area of research includes Mobile communication, SDR, Electronic welfare. Email: tapan.das@jecrcu.edu.in.

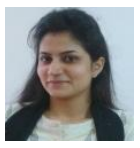

Yashaswini Sharma is pursuing her M.Tech in Embedded Systems in the department of Electronics and Communication Engineering at JECRC University, Jaipur, Rajasthan. She completed her B.Tech in Electronics and Communication. She attended and presented papers in National conferences. Email: yashaswini.sharma19@gmail.com. 